\title{
Current Indications for Carotid Endarterectomy
}

\author{
Christopher M. LOFTUS
}

\begin{abstract}
Department of Neurosurgery, The University of Oklahoma College of Medicine, Oklahoma City, Oklahoma, U.S.A.
\end{abstract}

\begin{abstract}
Four randomized cooperative trials for asymptomatic carotid artery disease and three randomized cooperative trials of symptomatic carotid artery disease have been completed and published. There are now firm and proven indications for carotid artery reconstruction. Asymptomatic carotid artery disease with $60 \%$ or greater linear stenosis on angiography has been shown to be better treated with surgery than with medical therapy alone. For symptomatic patients, linear stenoses of $50 \%$ or greater have been shown to have a significant benefit with surgical treatment. All surgical recommendations are based on a morbidity/mortality of $3 \%$ or less for the individual surgeon.
\end{abstract}

Key words: carotid artery diseases, carotid surgery, Asymptomatic Carotid Atherosclerosis Study, North American Symptomatic Carotid Endarterectomy Trial

\section{Introduction}

Carotid endarterectomy to prevent ischemic stroke in the distribution of a stenotic artery was introduced in 1954. ${ }^{111}$ Carotid circulation disease and its surgical correction are best studied when subdivided into asymptomatic and symptomatic forms. Asymptomatic carotid artery disease includes patients with asymptomatic carotid bruits, patients with symptoms referable to one carotid artery territory with radiographic demonstration of clinically silent contralateral carotid artery stenosis or ulceration, and patients who, while being prepared for major surgical procedures (most commonly coronary or peripheral vascular surgery), are found to have auscultatory or radiographic evidence of carotid pathology. I also include in the asymptomatic category patients with silent Hollenhorst plaques in the retina, ${ }^{16)}$ patients with silent cerebral infarcts, and patients with high-grade asymptomatic stenoses which are seen to be progressing on serial evaluations. Symptomatic carotid artery disease encompasses a spectrum of presentations, and includes classical transient ischemic attacks (TIAs) (usually amaurosis fugax, hemimotor or hemisensory deficits, and/or disturbances in speech, all lasting from a few seconds to several hours), reversible ischemic neurological deficits (which may present with the same symptoms but last up to 24 hours), crescendo TIAs (stereotypic TIAs which occur multiple times in a 24-hour period), stroke in evolution, and com- pleted stroke from carotid origin. Symptomatic carotid artery disease is also defined to include acute or subacute carotid artery occlusion (if neurological findings are present) as well as the so-called "stump syndromes."

Both asymptomatic and symptomatic carotid artery disease have been the subject of considerable debate in years past, and the literature dealing with surgical indications was confusing and often contradictory. It is gratifying to see that various cooperative trials have now given firm answers for both problems, and that surgery has been upheld as the cornerstone of therapy in patients with carotid artery stenosis. It is also important to understand, however, which patient groups were studied in the cooperative randomized trials, what conclusions can be drawn from the data, and which clinical questions were not studied, and thus remain unaddressed. Whereas a discussion of asymptomatic carotid artery disease involves primarily a comparison of operated patients versus unoperated control groups, any consideration of surgery for symptomatic carotid artery disease must be based on objective comparison of surgical morbidity and results with both the natural history of the disease process and the best available medical therapy. The surgical risk in elective carotid endarterectomies performed in major centers approaches $3 \%$, and this figure should be used for evaluation of therapeutic choices. Guidelines for acceptable surgical morbidity and mortality have recently been developed by both the American 
Heart Association Stroke Council ${ }^{5]}$ and the American College of Physicians. ${ }^{1)}$

\section{Cooperative Trials}

\section{Asymptomatic disease}

There have been four major completed prospective, randomized trials comparing medical and surgical therapies in patients with asymptomatic internal carotid artery (ICA) stenosis; the Carotid Artery Stenosis with Asymptomatic Narrowing Operation Versus Aspirin (CASANOVA) study, the Mayo Asymptomatic Carotid Endarterectomy Trial (MACE), the Veterans Administration Asymptomatic Surgical Trial (VAAST - VA CSP 167), and the Asymptomatic Carotid Atherosclerosis Study (ACAS), and there is one European trial, the Asymptomatic Carotid Surgery Trial which is continuing patient entry. Unlike symptomatic carotid artery disease, where all three cooperative trials came to essentially the same conclusion (that surgery was justified), the asymptomatic trials have yielded somewhat conflicting results.

All of the completed trials used percent angiographic linear stenosis of the cervical carotid artery as the entry criterion for surgery (it was possible to remain in the medical arm of the ACAS trial without angiography, but all surgical patients had angiograms performed). Ulceration and plaque morphology, while noted, did not enter into the randomization process. Thus the indications data currently validated by cooperative trials are based on linear stenosis only.

The first of these trials to be completed was the German CASANOVA study of 410 patients. This trial did not show any surgical benefit in reducing morbidity and mortality for patients with asymptomatic ICA stenosis of $<90 \%$. Medical treatment in this study consisted of aspirin and dipyridamole. No recommendations were made for patients with higher $(>90 \%)$ grades of stenosis, since such patients unfortunately were excluded from this trial $^{8,10)}$ The CASANOVA trial was methodologically flawed by allowing crossovers between medical and surgical groups for progression of stenosis under observation and for allowing empirical surgery in the face of known contralateral occlusion (patients with $>90 \%$ stenosis were excluded from this trial on the basis of presumed surgical benefit), and because of these the data have been somewhat discounted as later trials became available.

In the Mayo Clinic MACE study, which was published in 1992,71 patients with a $50-99 \%$ carotid artery stenosis were randomized to $80 \mathrm{mg} /$ day of aspirin or endarterectomy. Aspirin use was discouraged in the surgical arm. This trial was terminated early because of the frequency of heart attacks in the surgical group not receiving aspirin. No conclusions on the frequency of cerebral ischemic events were made, and no surgical benefit could be demonstrated in this small study. If anything, findings from this trial pointed to the benefit of prophylactic aspirin in preventing myocardial infarction in patients having carotid endarterectomy. ${ }^{25)}$

The VAAST (VA CSP 167) trial randomized 444 male patients in 1987. Patients in the medical arm of this study received $325 \mathrm{mg}$ of aspirin twice daily. Findings from this trial showed that carotid endarterectomy reduced the overall incidence of ipsilateral neurological events (TIAs included) for patients with asymptomatic ICA stenosis of $50 \%$ or more. However, this trial found no significant effect from carotid endarterectomy on the combined incidence of stroke and death, and thus no surgical benefit was conferred for these endpoints. ${ }^{15)}$ Whereas some argued that the VAAST provided justification for surgery on asymptomatic lesions, many others felt that the demonstrated reduction of only TIAs, without an impact on stroke or death was not sufficient justification for prophylactic endarterectomy, since the onset of TIAs in previously asymptomatic patients would customarily trigger carotid artery reconstruction, especially in patients with highgrade stenosis. ${ }^{4}$

The largest of these asymptomatic trials, ACAS, as mentioned above, reached a stopping rule on September 16, 1994, and the data was released to the coinvestigators. ACAS randomized 1659 patients at 39 centers (834 patients treated medically and 825 patients treated surgically) between the ages of 40 to 79 years with a $60-99 \%$ ICA stenosis to $325 \mathrm{mg} /$ day of aspirin plus risk factor management or endarterectomy plus medical therapy. Angiogram confirmation was not absolutely necessary, except for the surgical group, as ultrasound criteria could be used to meet inclusion criteria. The primary outcome measure was any stroke or death in the perioperative period and any ipsilateral stroke thereafter. After a median follow-up of 2.7 years, the aggregate estimated risk over 5 years for ipsilateral stroke or perioperative stroke or death was $5.1 \%$ for patients who underwent surgery and $11.0 \%$ for patients treated medically. Carotid endarterectomy reduced the estimated 5-year risk for ipsilateral stroke by $1 \%$ per year, but major ipsilateral stroke or perioperative death was not significantly reduced. The benefit likewise was shown for the overall study group and for males but could not be statistically demonstrated when females alone were analyzed as a subgroup. The success of the operation depended on a perioperative morbidity and mortality of $<3 \%$. It is esti- 
mated that approximately 19 carotid endarterectomies would need to be performed to prevent one stroke. ${ }^{13)}$

It is important to realize that all patients with a $60-$ $99 \%$ stenosis were analyzed together in the ACAS study. ACAS was thus not able to assign a higher risk to higher degrees of stenosis (this has been proven by North American Symptomatic Carotid Endarterectomy Trial [NASCET] for symptomatic patients, as will be seen below). The degree of carotid artery stenosis question was evaluated by the European Carotid Surgery Trialists (ECST) Collaborative Group, ${ }^{12)}$ who presented data on contralateral asymptomatic stenosis gathered from their patients who had randomization of the symptomatic side (ECST is a randomized symptomatic carotid trial, to be discussed below). Using data from 2295 patients in the ECST trial the investigators determined that the overall Kaplan-Meier estimate of stroke risk at 3 years was $2.1 \%$ in the distribution of the asymptomatic ICA. More striking was that patients in each decile up to $70 \%$ stenosis of the asymptomatic ICA had a very low risk of stroke $(<2 \%)$. The risk jumped to $5.7 \%$ in those patients with a $70-99 \%$ stenosis, although this was not statistically significant. Also, the stroke risk was $9.8 \%$ in the $80-$ $89 \%$ ICA stenosis decile and increased to $14.4 \%$ in patients with $90-99 \%$ asymptomatic ICA stenosis. They concluded that population screening could not be justified given the overall low risk of stroke in the distribution of an asymptomatic ICA. I would argue somewhat with this strong conclusion, as will be seen later when my personal preferences are discussed. (Recall that the stroke event rate in the ACAS study population was too small to analyze by decile of stenosis.) It should be kept in mind that this data cannot be directly compared to the ACAS data for several reasons. First, all arteries opposite to the asymptomatic side were symptomatic in the ECST trial. Second, the degree of stenosis was measured differently in these two trials. Third, the ECST second side data is not randomized data. Finally, the ACAS study had a median follow-up of 2.7 years while the ECST study had a mean follow-up of 4.5 years.

In conclusion, several excellent studies addressing the usefulness of carotid endarterectomy in patients with asymptomatic ICA stenosis have been done in the last few years. Early, small, and probably flawed studies did not show a benefit from surgical reconstruction. The largest of the trials, the ACAS study, showed that endarterectomy was beneficial in asymptomatic patients with $60-99 \%$ ICA stenosis. The ACAS trial did not have sufficient statistical power to show a benefit stratified by degree of stenosis nor for females, perhaps because the trial was stopped prematurely by the monitoring committee (it was felt to be unethical to continue the trial when the overall group was seen to have a statistically evident benefit from surgery over medical treatment). A randomized European Asymptomatic Carotid Surgery Trial is currently underway, ${ }^{14)}$ but it is likely that the few unanswered questions not addressed by ACAS will remain so.

\section{Symptomatic disease}

There were three major trials for symptomatic carotid artery disease, all reaching the same conclusion (that surgery had a statistical benefit over medical therapy for carotid artery stenosis of $70 \%$ or more). The NASCET was published in $1991 .^{29,32)} \mathrm{A}$ total of 659 patients who had a carotid TIA or nondisabling stroke within the preceding 120 days were randomized to surgery or optimal medical therapy including antiplatelet treatment with aspirin. They were required to have an angiogram-proven $70-99 \%$ stenosis of the corresponding ICA. While the surgical patients had slightly more strokes or deaths in the 30-day perioperative period, their overall risk of stroke or death at 2 years was significantly reduced. For different endpoints, absolute risk reduction in favor of surgery were as follows: 1) $17 \%$ for ipsilateral stroke, 2) $15 \%$ for all strokes, 3) $16.5 \%$ for the combined outcome of all strokes and death, 4) $10.6 \%$ for major ipsilateral stroke, 5) $9.4 \%$ for all major strokes, and 6) $10.1 \%$ for major stroke and death. Unlike the ACAS data, the risk of ipsilateral stroke increased with the extent of carotid artery stenosis. The endarterectomies were performed by experienced surgeons with an overall perioperative stroke and death rate of less than $6 \%$. The results for "moderate" stenosis of $30-69 \%$ are now also available from NASCET. A surgical benefit has been shown for symptomatic males with $\geq 50 \%$ linear stenosis.

The ECST trial was also published in $1991{ }^{28)}$ It too compared carotid endarterectomy to best medical therapy in 1152 patients with carotid TIA or nondisabling strokes within 6 months from inclusion. The degree of stenosis was measured differently than NASCET (the NASCET measurement system was more rigorous). Again, patients with $70 \%$ to $99 \%$ stenosis who underwent surgery had significantly fewer strokes or deaths. The ECST also indicated that patients with a "mild" stenosis of $0-29 \%$ or "moderate" stenosis of $30-69 \%$ had no benefit from carotid endarterectomy. The failure of ECST to show a benefit for "moderate" stenosis is provocative, but since the NASCET measurement system is more stringent, it is possible (and indeed has been report ed) that ECST patients in the $30-69 \%$ group would 
not even qualify for inclusion in the NASCET trial. The final answer for moderate stenosis will wait until NASCET data is available.

The Veterans Administration Symptomatic Surgical Trial - VA CSP 309 evaluated 189 males with carotid TIA or minor strokes within 120 days before inclusion and an associated angiographic stenosis of $>50 \% .{ }^{24 l}$ The study was stopped prematurely when the NASCET and ECST results were released; it was felt to be unethical to continue randomization in light of the surgical benefit demonstrated by the larger trials. All patients received $325 \mathrm{mg}$ of aspirin per day. At a mean follow-up of 12 months, the patients who had surgery had significantly fewer ipsilateral TIAs and strokes. The benefit of surgery was greater in patients with greater than $70 \%$ ICA stenosis. This third smaller study thus reinforced the conclusions of the aforementioned trials.

In summary, carotid endarterectomy is highly beneficial for symptomatic patients with recent, nondisabling carotid artery ischemic events and ipsilateral $50 \%$ to $99 \%$ carotid artery stenosis. Carotid endarterectomy is not beneficial for symptomatic patients with $0 \%$ to $49 \%$ stenosis. At present I continue to offer surgery to such patients.

\section{Special Issues Not Addressed by the Randomized Trials}

\section{Critical stenosis}

A subset of the asymptomatic bruit/stenosis population was felt by many neurovascular surgeons to represent a high-risk group for acute carotid artery occlusion with neurological catastrophe. These were patients who had either an initial $80 \%$ or greater stenosis documented by non-invasive studies, angiography or magnetic resonance angiography, or patients with lesser degrees of stenosis, who were followed with serial examinations and who then progressed to develop an $80 \%$ or greater lesion. The optimal management of these "critical" high-grade asymptomatic stenoses was greatly debated. Hemodynamic studies have indicated that critical reductions in cerebral blood flow may not be reached until $75-84 \%$ diameter stenosis has occurred, indicating that stenosis must be of a very high grade to be significant. ${ }^{2)}$ Ojemann et al. ${ }^{33)}$ believed that a $2 \mathrm{~mm}$ residual angiographic lumen represented a $70 \%$ narrowing and that carotid artery reconstruction was justified. Roederer et al. ${ }^{35)}$ studied prospectively 167 asymptomatic bruit patients who were followed with duplex scans at 6 -month to 1-year intervals. Smoking, diabetes mellitus, and age $<65$ years were identified as major risk factors for progression of silent stenosis. Ten patients suffered subsequent neurological events (all ipsilateral to the carotid bruit), and $90 \%$ of these were in patients who had an $80 \%$ or greater stenosis. The authors felt that this high degree of correlation between progression of stenosis and the onset of clinical events justified serial scanning to monitor the progression of carotid artery stenosis, and that $80 \%$ or greater lesions should be reconstructed. Moneta et al. ${ }^{27)}$ studied 129 asymptomatic high-grade $(80-99 \%)$ stenosis patients, of which 56 underwent endarterectomy and 73 were followed. There was one perioperative stroke $(1.8 \%)$ and nine strokes (all unwarned; five from carotid artery occlusion) in the unoperated group. Their data provided convincing evidence that surgery may be protective in these high-grade stenosis patients. Based on these reports many surgeons felt justified in correcting such severe but otherwise asymptomatic lesions.

Chambers and Norris, ${ }^{97}$ however, reported that in asymptomatic patients with stenosis of all degrees the risk of cardiac ischemia was higher than that of stroke. In their series, although the risk of cerebral ischemic events was highest in patients with severe carotid artery stenosis, in most instances these patients did not have strokes without some sort of warning event, and even those patients who progressed to complete occlusion while being observed had a relatively benign outcome. Bogousslavsky et al. ${ }^{6}$ had likewise followed 38 patients with $90 \%$ or greater asymptomatic stenosis for a mean period of 48 months. This patient group with severe stenosis had a $1.7 \%$ annual rate of unwarned stroke, sufficiently low that the authors could not justify prophylactic endarterectomy. Most recently Norris et al $^{311}$ followed 696 patients for a mean of 41 months with non-invasive studies. While the combined TIA/stroke rate for patients with $>75 \%$ stenosis was a significant $10.5 \%$, the ipsilateral stroke rate (without warning TIA) was $2.5 \%$ for patients with $>75 \%$ stenosis, and with $<75 \%$ stenosis $1.1 \%$. This data re-emphasized the need for surgical action in symptomatic high-grade stenosis patients, but did not identify a high-risk asymptomatic subgroup. Many reviews of this subject ${ }^{26,38)}$ recommended medical management of the patient with asymptomatic carotid bruit or stenosis with antiplatelet aggregating therapy and attention to contributing risk factors (hypertension), with surgical intervention deferred until such time as frank TIAs develop. Others, like Moneta et al., ${ }^{27)}$ felt justified in prophylactically reconstructing the $75-80 \%$ or greater lesion. In the opinion of this author, ACAS data has essentially resolved this conflict in favor of surgery. 


\section{Silent cerebral infarction}

There had been some evidence to suggest that silent small cerebral infarction (seen on computed tomography [CT]] may justify carotid endarterectomy in otherwise asymptomatic patients. Norris and $\mathrm{Zhu}^{30)}$ compared CT scans with carotid Doppler in patients identified to have asymptomatic carotid artery stenosis. They reported ipsilateral small $(<15$ $\mathrm{mm}$ ) silent infarcts in $10 \%$ of mild (35-50\%), $17 \%$ of moderate $(50-75 \%)$, and $30 \%$ of severe $(>75 \%)$ stenosis patients. They concluded that silent cerebral infarction may be an indication for ipsilateral carotid endarterectomy, particularly in severe stenosis patients. In light of the ACAS trial data now available (and mentioned earlier), I agree that such lesions should be reconstructed if $\geq 60 \%$ stenosis exists, and I feel that the gray area will now be the management of patients with $<60 \%$ stenosis and silent cerebral ipsilateral infarction.

\section{Hollenhorst plaque}

It is important to distinguish symptomatic retinal plaques from asymptomatic ones. ${ }^{16)}$ Multiple authors have reported plaques associated with either amaurosis fugax or retinal artery occlusion with visual field deficits and I have little doubt that these represent symptomatic carotid artery lesions. ${ }^{3,36,37)}$ These symptomatic lesions certainly deserve active investigation for carotid origin embolization.

In 1973, Hollenhorst's group reported on 208 consecutive patients observed to have retinal cholesterol emboli who had been followed for at least 6 years. ${ }^{34)}$ This group was mixed and many of them had visual symptoms associated with their cholesterol emboli. This group of patients had significantly decreased survival compared to a heterogeneous comparison group with a survival rate $13 \%$ less than expected in the first year increasing to $80 \%$ less than expected by the eighth year of observation. The cause of death in many of these patients was related to diffuse vascular disease with myocardial infarction being the greatest factor. Hollenhorst once again concluded on the basis of this data that these plaques warrant aggressive cardiac and cerebrovascular investigation.

Patients with truly asymptomatic retinal cholesterol emboli represent a much more unusual and smaller group. Very little data is available as to the natural history and prognosis of these patients. Bruno et al. ${ }^{7)}$ recently studied 70 consecutive males with asymptomatic retinal cholesterol emboli and compared them to a control group of 21 randomly selected subjects without retinal emboli. Patients in their study group had a significantly higher prevalence of hypertension and smoking history than did the control group. The prevalence of carotid artery stenosis $\geq 50 \%$ ipsilateral embolus was only $13 \%$, however, and this was not significantly different from that in control subjects. However, carotid artery stenosis on either side $\geq 50 \%$ was more common in patients with asymptomatic retinal cholesterol emboli. According to Bruno's data, asymptomatic retinal cholesterol emboli did indicate a higher prevalence of systemic vascular disease and of ischemic heart disease, similar to that reported by Pfaffenbach and Hollenhorst. ${ }^{34)}$ Their data, however, did not support the concept that asymptomatic retinal cholesterol emboli are the harbingers of cerebrovascular events or of the presence of an unstable carotid atherosclerotic plaque. In keeping with this, it should be noted that asymptomatic retinal cholesterol emboli were not considered entry criteria for the NASCET carotid endarterectomy trial.

What then can be said about the presence of Hollenhorst plaques? Certainly any identifiable retinal lesion with visual symptoms must be considered a symptomatic carotid event until proven otherwise and warrants full investigation. I have performed carotid endarterectomy on many patients whose initial presentation was visual loss from central retinal artery occlusion by an embolic plug. The significance of asymptomatic lesions in the retina is confusing and not well studied at present. Available data would indicate that these do not represent a high-risk group but it had been my inclination to investigate these patients actively regardless on the few occasions when absolutely no visual symptoms can be elicited and of course I now recommend carotid endarterectomy for any such patients found to have $\geq 60 \%$ stenosis.

\section{Clinical Approach to the Carotid Patient}

Patients present to the cerebrovascular surgeon in a number of different ways. Asymptomatic patients are customarily elective and seen in the office or clinic, and are most often sent by primary care physicians for a surgical opinion as well as surgical treatment. They may or may not have had non-invasive carotid studies performed, but most likely will not yet have undergone angiography. Symptomatic patients are more likely to be first seen in hospital in consultation, and are often heparinized.

My diagnostic plan for asymptomatic patients differs from that for patients with neurological symptoms. ${ }^{17-23)}$ I proceed first with non-invasive (duplex) scanning to ascertain the degree of stenosis present (whereas in symptomatic patients I usually go straight to angiography). The quality of the non-in- 
vasive laboratory is crucial at this step, and I prefer to use a validated and standardized laboratory with which I am familiar. If the stenosis is found to be $60 \%$ or greater, I proceed with a rapid workup with the aim of performing surgery as soon as possible. It is my preference to have formal and complete angiography in every case including selective injection of both carotid and both vertebral arteries. Attention to intracranial cross filling is also important and I insist on biplane cervical angiography in every case so that we can determine the relationship of the ICA and external carotid artery. When digital angiography is performed, as is common in my center now, I ask that the arterial image be superimposed on a bone image so that I can ascertain the height of the carotid bulb in relationship to the cervical spine, the angle of the mandible, and the hyoid bone.

There is at present a considerable degree of interest in operating on patients based on magnetic resonance angiography alone. This has not been my practice as yet but clearly as the technology develops it may become more prevalent. I have not operated on patients on the basis of duplex scanning alone and do not imagine I will do so in the future.

Patients who are seen for consideration of carotid artery surgery are customarily on aspirin or some form of anticoagulation, but if not they are started on aspirin at the time of first visit. I do not stop preoperative antiplatelet therapy in preparation for surgery. Heparinized patients (usually with crescendo TIAs) are maintained on full anticoagulation up to and throughout the surgical procedure.

The remainder of the preoperative evaluation is uneventful. A careful neurological examination is performed and a history is carefully obtained to be certain that neurological symptoms have not been overlooked. Any history of cardiac disease warrants cardiology consultation and patients who are smokers are advised to cease smoking as far as possible in advance of the surgical procedure.

\section{Conclusions}

It was previously very difficult for me to sort out the complexities of historical and non-randomized trials and to determine which, if any asymptomatic patients required surgery. At the risk of seeming oversimplified, this problem has essentially been solved. I recommend surgery to any asymptomatic patient with linear carotid artery stenosis of $\geq 60 \%$, and for symptomatic patients with linear stenosis of $\geq 50 \%$ by NASCET criteria. $^{22\}}$

There are still problems with this approach. It assumes that I have ignored the data from CASANOVA, MACE, VA CSP 167, and the count- less non-randomized trials which could not show a surgical benefit in asymptomatic patients. This is essentially what I have done, putting my trust in ACAS, which I feel is the largest and most scientifically valid trial.

There is, and will continue to be, much room for debate among neurologists and neurosurgeons regarding the indications for carotid artery reconstruction. In my opinion, however, the ACAS and NASCET data will stand the test of time just as the extracranial-intracranial bypass data has done, and will permanently reshape practice patterns for carotid endarterectomy in much the same fashion.

\section{References}

1) American College of Physicians: Indications for carotid endarterectomy. Ann Intern Med 111: 675677,1989

2) Archie JP, Feldtman RW: Critical stenosis of the internal carotid artery. Surgery 1: 67-72, 1981

3) Balla JI, Howat ML, Walton JN: Cholesterol emboli in retinal arteries. J Neurol Neurosurg Psychiatry 27: $144-148,1964$

4) Barnett HJM, Haines SJ: Carotid endarterectomy for asymptomatic carotid stenosis. $N$ Engl J Med 328: 276-279, 1993

5) Beebe HG, Clagett GP, De Weese JA, Moore WS, Robertson JT, Sandok B, Wolf PA: Assessing risk associated with carotid endarterectomy. A statement for health professionals by an Ad Hoc Committee on Carotid Surgery Standards of the Stroke Council, American Heart Association. Circulation 79: 472473, 1989

6) Bogousslavsky J, Despland PA, Regli F: Asymptomatic tight stenosis of the internal carotid artery: Longterm prognosis. Neurology 36: 861-863, 1986

7) Bruno A, Russell PW, Jones WL, Austin JK, Weinstein ES, Steel SR: Concomitants of asymptomatic retinal cholesterol emboli. Stroke 23: 900-902, 1992

8) CASANOVA Study Group: Carotid surgery versus medical therapy in asymptomatic carotid stenosis. Stroke 22: 1229-1235, 1991

9) Chambers BR, Norris JW: Outcome in patients with asymptomatic neck bruits. $N$ Engl J Med 315: 860865,1986

10) Diener HC, Hamann $H$, Schäfer $H$, Paulat $K$, the CASANOVA Study Group: Carotid surgery versus medical therapy in asymptomatic carotid stenosis. Neurology 40 (Suppl 1): 415, 1990

11) Eastcott HHG, Pickering GW, Rob CG: Reconstruction of internal carotid artery in a patient with intermittent attacks of hemiplegia. Lancet 2: 944-946, 1954

12) European Carotid Surgery Trialists Collaborative Group: Risk of stroke in the distribution of an asymptomatic carotid artery. Lancet 345: 209-212, 1995 
13) Executive Committee for the Asymptomatic Carotid Atherosclerosis Study: Endarterectomy for asymptomatic carotid artery stenosis. JAMA 273: 1421-1428, 1995

14) Halliday AW: The Asymptomatic Carotid Surgery Trial (ACST): Rationale and design. Eur J Vasc Surg 8: 703-710, 1994

15) Hobson RW 2d, Weiss DG, Fields WS, Goldstone J, Moore WS, Towne JB, Wright CB: Efficacy of carotid endarterectomy for asymptomatic carotid stenosis. $N$ Engl J Med 328: 221-227, 1993

16) Hollenhorst RW: Significance of bright plaques in the retinal arterioles. JAMA 178: 123-129, 1961

17) Loftus CM: Surgical management options to prevent ischemic stroke, in Adams HP (ed): Handbook of Cerebrovascular Diseases. New York, Marcel Dekker, 1993, pp 315-358

18) Loftus CM: Carotid Endarterectomy: Principles and Technique. St Louis, Quality Medical Publishing, 1995, pp 1-242

19) Loftus CM: Carotid endarterectomy (guest editor). Techniques in Neurosurgery 3: 1-84, 1997

20) Loftus CM: Acute surgical therapeutic interventions in stroke patients, in Selman WR, Lust WD (eds): Brain Attack: The Acute Pathophysiology of Cerebral Ischemia and Its Emergent Treatment. Neurosurg Clin N Am 8: 227-236, 1997

21) Loftus CM: Carotid endarterectomy: How the operation is done. Clin Neurosurg 44: 243-265, 1997

22) Loftus CM, Hopkins LN: Paradoxical indications for carotid endarterectomy. Neurosurgery 36: 99-100, 1995

23) Loftus CM, Quest DO: Technical controversies in carotid artery surgery. Neurosurgery 20: 490-495, 1987

24) Mayberg MR, Wilson SE, Yatsu F, Weiss DG, Messina L, Hershey LA, Colling C, Eskridge J, Deykin D, Winn HR: Carotid endarterectomy and prevention of cerebral ischemia in symptomatic carotid stenosis. JAMA 266: 3289-3294, 1991

25) Mayo Asymptomatic Carotid Endarterectomy Study Group: Results of a randomized controlled trial of carotid endarterectomy for asymptomatic carotid stenosis. Mayo Clin Proc 67: 513-518, 1992

26) Mohr JP: Asymptomatic carotid artery disease. Stroke 13: 431-433, 1982

27) Moneta GL, Taylor DC, Nicholls SC, Bergelin RO, Zierler RE, Kazmers A, Clowes AW, Strandness DE $\mathrm{J} r$ : Operative versus nonoperative management of asymptomatic high-grade internal carotid artery stenosis: improved results with endarterectomy. Stroke 18: $1005-1010,1987$

28) MRC European Carotid Surgery Trial: Interim results for symptomatic patients with severe (70$99 \%)$ or with mild $(0-29 \%)$ carotid stenosis. Lancet 337: 1235-1243, 1991

29) NASCET Investigators: Clinical alert. Benefit of carotid endarterectomy for patients with high-grade stenosis of the internal carotid artery. National Institute of Neurological Disorders and Stroke. February 25, 1991

30) Norris JW, Zhu CZ: Silent stroke and carotid stenosis. Stroke 23: 483-485, 1992

31) Norris JW, Zhu CZ, Bornstein NM, Chambers BR: Vascular risks of asymptomatic carotid stenosis. Stroke 22: 1485-1490, 1991

32) North American Symptomatic Carotid Endarterectomy Trial Collaborators: Beneficial effect of carotid endarterectomy in symptomatic patients with high grade carotid stenosis. N Engl J Med 325: 445-453, 1991

33) Ojemann RG, Crowell RM, Roberson GH, Fisher CM: Surgical treatment of extracranial carotid occlusive disease. Clin Neurosurg 22: 214-263, 1975

34) Pfaffenbach DD, Hollenhorst RW: Morbidity and survivorship of patients with embolic cholesterol crystals in the ocular fundus. Am J Ophthalmol 75: 66-72, 1973

35) Roederer GO, Langlois YE, Jager KA, Primozich JF, Beach KW, Phillips DJ, Strandness DE Jr: The natural history of carotid arterial disease in asymptomatic patients with cervical bruits. Stroke 15: 605-613, 1984

36) Russell RW: Atheromatous retinal embolism. Lancet 2: 1354-1356, 1963

37) Russell RW: The source of retinal emboli. Lancet 2: 789-792, 1968

38) Yatsu FM, Hart RG: Asymptomatic carotid bruit and stenosis: A reappraisal. Stroke 14: 301-304, 1983

Address reprint requests to: C. M. Loftus, M.D., Department of Neurosurgery, The University of Oklahoma College of Medicine, 711 Stanton L. Young Boulevard, Suite 206, Oklahoma City, OK 73104, U.S.A. 